\title{
Choroid plexus carcinoma: A case report and literature review
}

\author{
Azhani C' ${ }^{1}$ Chan KH ${ }^{1}$, Fadli $M^{2}$ and Saufi $A^{* 1}$ \\ ${ }^{1}$ Department of Neurosurgery, Kulliyyah of Medicine, International Islamic University Malaysia \\ ${ }^{2}$ Department of Pathology, Hospital Tengku Ampuan Afzan, Malaysia
}

\begin{abstract}
Choroid plexus carcinoma is a rare tumor representing less than $1 \%$ of all brain tumors. In adult, the incidence is extremely rare making the diagnosis difficult. Majority of choroid plexus tumor is found in the ventricle. However, ectopic sites such as intracranial extraventricular or spine have been reported. We report a case of choroid plexus carcinoma in a 39-year-old man. The clinical presentation, pathology and management are discussed.
\end{abstract}

\section{Introduction}

Choroid plexus carcinoma is a rare tumor especially in adult. In total population, choroid plexus tumor only represents less than $1 \%$ of all brain tumors [1]. Out of all choroid plexus tumors, only $15-30 \%$ is carcinoma. In adult however, due to its extremely rare occurrence, the diagnosis of choroid plexus carcinoma should be made with caution as it more frequently resembles a metastatic papillary tumor such as from kidney and thyroid [2].

Most of choroid plexus tumor arises in intraventricular, but cases have been reported as arising from ectopic sites such as intracranial but extraventricular and also in spinal canal without intracranial lesion [212]. It has been suggested in a few literatures that for older children and adult, a gross total resection is the best method of treatment followed by adjuvant chemotherapy and radiotherapy $[1,3,4]$. However, surgical resection poses a challenge as it frequently intensely vascular tumor, leading to extreme and heavy bleeding during surgery, thus affecting patients' survival [2]. This case report highlights the extremely rare case of adult choroid plexus carcinoma, arising from basal ganglia. The clinical presentation, pathology and management are well discussed.

\section{Case summary}

We report the case of a 39-year-old man presented with reduced conscious level for 3 days prior to admission preceded by two weeks history of headache and progressive left sided weakness. His wife gave a history of facial asymmetry two months earlier. At the time of admission, his Glasgow Coma Scale (GCS) was 10/15 (E4V1M5). Pupils were bilaterally equal and reactive. Papilledema was present bilaterally Left-side hemiparesis (MRC grade 1/5) with left upper motor neuron facial paresis was present.

Computed Tomography (CT) scan of brain showed a large heterogenous density mass in right temporoparietal region measuring $5.2 \times 5.2 \times 4.8 \mathrm{~cm}$ with perilesional edema (Figure 1 ). The mass was compressing the ipsilateral lateral and third ventricle with a midline shift of $0.9 \mathrm{~cm}$. The contralateral left lateral ventricle and temporal horn of right lateral ventricle were dilated. Magnetic Resonance Angiograpy (MRI) brain revealed heterogenous intra axial right basal ganglia mass (Figure 2). The mass was hypointense on T1, heterogeneously hyperintense on T2 and enhanced post Gadolinium. There was also small hypointense area seen at superior part of the mass that may represent micro bleeding. The right lateral ventricle was compressed and contralateral lateral ventricle was dilated. There was midline shift present. The right middle cerebral artery was displaced anteriorly but vessels were patent and normal in caliber. The mass also was significantly vascular. Based on radiology, a diagnosis of vascular high grade tumor such as GBM was kept.

He underwent right craniotomy and debulking of tumor through a transsylvian. Intraoperatively, the tumor was fragile, soft and highly vascular, consistent with features of high grade tumor as in GBM. There was feeding vessel from the lenticulostriate artery. Partial resection was done in view of intra-operative bleeding and tensed brain which limits further dissection. Post-operatively, he was kept sedated. Unfortunately, he deteriorated two weeks after surgery and succumbed to death.

Histopathology examination was reported as choroid plexus carcinoma with tumor exhibiting papillary pattern with central fibrovascular cone lined by cuboidal to columnar to pseudostratified epithelium. There were abundant mitotic figure and tumor necrosis. Immunohistochemistry stains positive for epithelial membrane antigen (EMA) (Figure 3).

\section{Discussion}

Adult brain tumors are more commonly secondary than primary brain tumors. Primary brain tumors comprises about $1.6 \%$ of all tumors diagnosed and of these Choroid Plexus Tumor (CPT) represents only less than $1 \%[1,4,11]$. Choroid Plexus Carcinoma (CPC) is less

Correspondence to: Mohamed Saufi Awang, MBBS (Adelaide), M Surg (Neurosurgery/USM), Associate Professor, Department of Neurosurgery, International Islamic University Malaysia, Kuantan, Malaysia, Tel: 09-5706171, Fax: 09-5146090, E-mail: saufiawang@iium.edu.my

Key words: choroid plexus carcinoma, basal ganglia, brain tumor

Received: April 05, 2017; Accepted: April 21, 2017; Published: April 24, 2017 


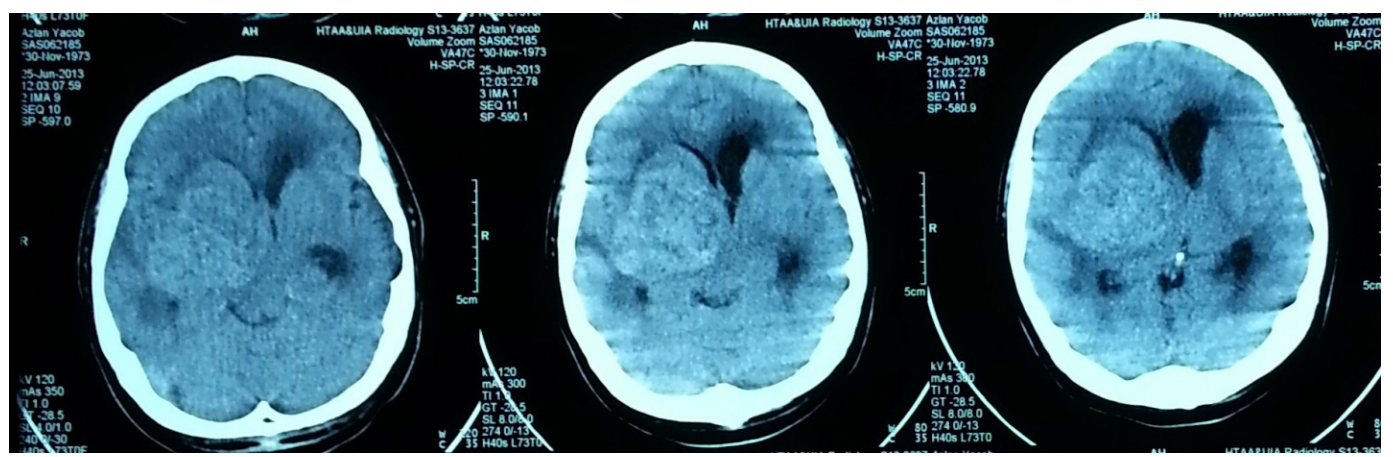

Figure 1: Pre-operative CT brain (non contrast).
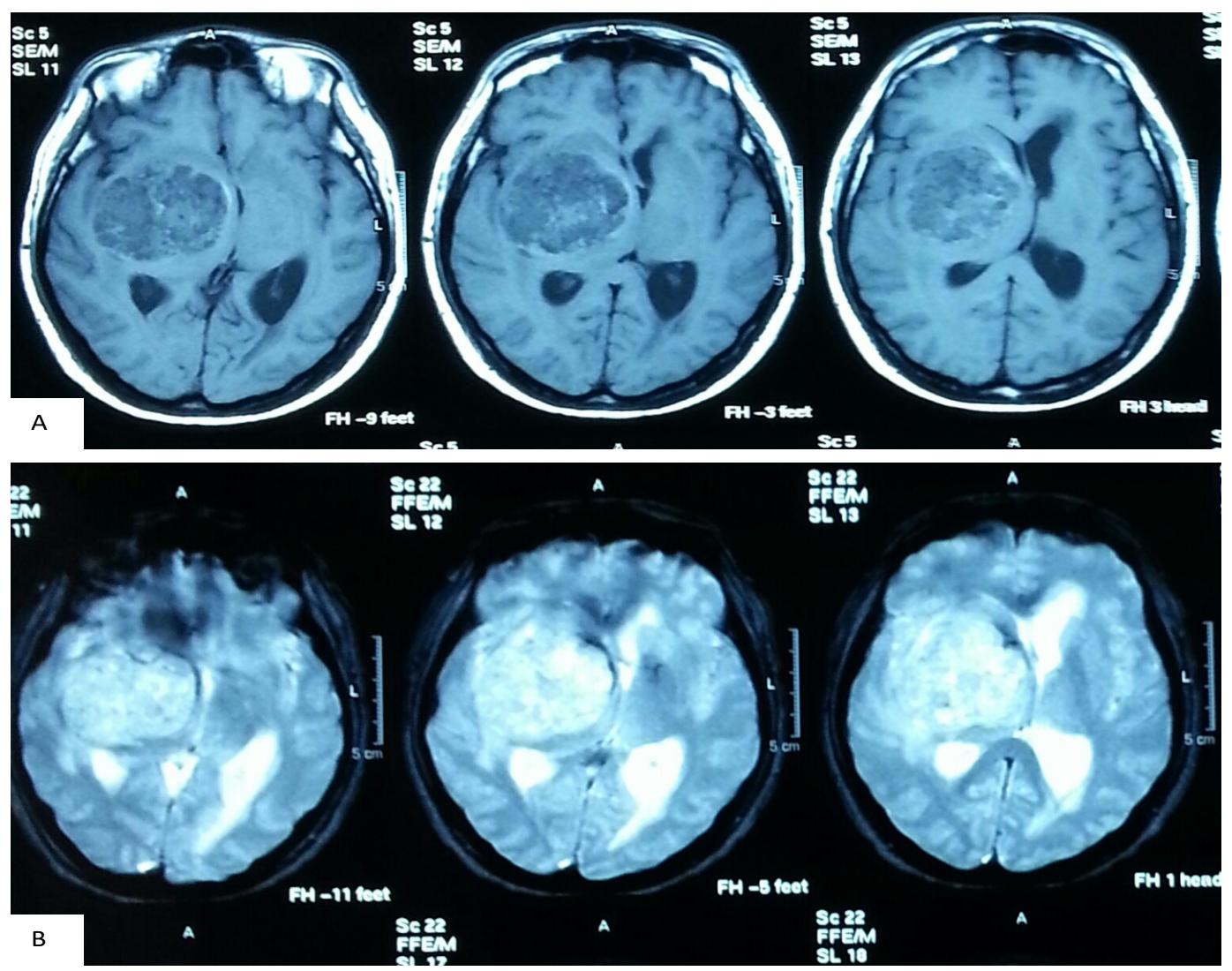

Figure 2: Pre-operative MRI. T1 weighted with Gado (a) and T2 weighted (b) images.

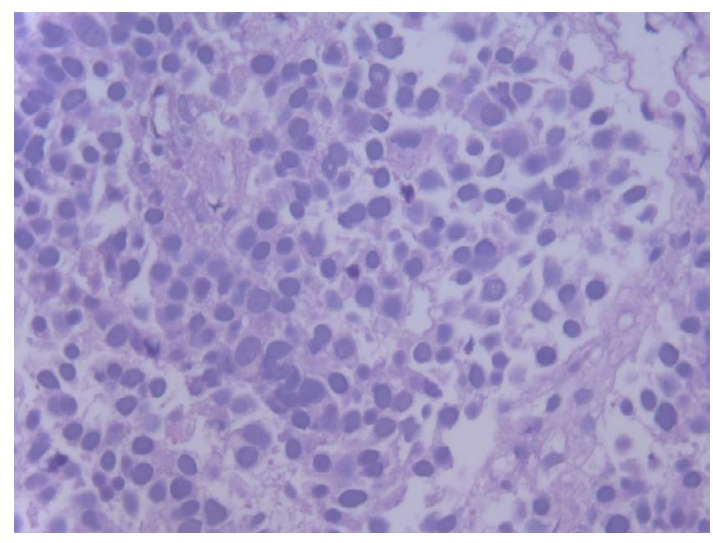

Figure 3: Histologic characteristic of resected tumor. Solid growth of carcinoma with focal papillary structures. H\&E magnification 190x. common than papilloma; estimated around $15-30 \%$ of choroid plexus tumors [11]. Sampath, et al. conducted a 20 year retrospective reviewed cases of CPT and found only 47 cases in total population, with $95 \%$ out of 19 cases of adult CPT are choroid plexus papiloma [12]. Most of the diagnosis of choroid plexus carcinoma is made in paediatrics age group and rarely reported in adult. If present in adult, it should be interpreted with caution and patient should be investigated thoroughly to exclude metastatic tumors particularly from renal and thyroid $[1,11]$. The median age of presentation is 3 years (ranging from 0 to 72 years old) with the youngest case recorded was an unusual congenital choroid plexus carcinoma detected antenatally at 29 weeks in utero $[3,8]$. In adult population, median age of CPT is 30 years and diagnosis of choroid plexus carcinoma is rarely made [12]. Choroid plexus papilloma is graded as WHO grade I tumor, whereas choroid plexus carcinoma is WHO grade III. 
The location of CPT are usually intraventricular; however extraventricular has been reported in several literatures [2,5,6,12]. Sampath et al also have found that CPT at ectopic site occurred only in adults according to their review [12]. Sites that have been reported to be ectopic include suprasellar region, foramen magnum, cerebellopontine angle and in the spinal canal in the absence of intracranial lesion. As choroid plexus tumors arise from epithelium of the choroid plexus, possible plausible theory for occurrence of extraventricular tumors is that it could either arise from dissemination through cerebrospinal fluid or presence of small tuft of choroid plexus extending from the Foramen of Luschka (in cerebellopontine angle choroid plexus carcinoma) [5]. Commonest site for adult is in fourth ventricle (63\%), while in paediatric age group is lateral ventricle (72\%) [12]. $12 \%$ of patients with CPC estimated to have metastasis at presentation with the frequency is higher in supratentorial lesion [3].

The presentations of symptoms vary and fall into 2 major categories which are intracranial hypertension and focal neurological deficit. Menon, et al. reviewed 25 cases of CPT and $72 \%$ of their patients presented with increase intracranial pressure symptoms, whereas Sampath noted $90 \%$ of patients in both adult and paediatric populations presented with hydrocephalus $[9,11]$. Most common focal neurological deficit sustained is cranial nerve VI palsy attributed to hydrocephalus. Other forms of neurodeficit include hemiparesis and sensory dysfunction. In a reported case of cerebellopontine angle CPT, other cranial nerves will also involve. Most of the symptoms contributed by the mass effect and hydrocephalus (both communicating and obstructive), but cases with primary bleeding from the tumor has been reported and markedly in tumor at ectopic area [2]. Intratumoral hemorrhage in a recurrent tumor has also been reported needing evacuation [10].

In investigating the tumor, both CT scan and MRI is needed, and sometimes angiography will be useful especially if we are suspecting a highly vascularized tumor. In CT scan, $75 \%$ of tumors will show heterogenous density, and 10-25\% will have calcifications [2]. MRI will usually show isointense on T1 image and heterogenous hyperintese on T2 with enhancement post Gadolinium contrast. Angiography can sometimes identify tumor blush which suggest presence of blood supply to the tumor. In Menon et al review, 18 of 25 patients have tumor blush present. If feeding vessel is identified, endovascular embolization has been attempted previously but with low success rate in view of tortuous tumor vessel and difficulty in cannulating the choroidal arteries. Further investigation will include searching for exclusion of primary tumor in suspicion of metastatic papillary growth in adult diagnosed with choroid plexus carcinoma. Extensive search of primary will include doing CT scan of thorax, abdomen and pelvis and also mammography. Most common primary resembling choroid plexus carcinoma includes renal cell carcinoma, thyroid malignancies and esophageal cancer. Thus history and clinical examination should be directed towards excluding these primaries as choroid plexus carcinoma is extremely rare in adult. Other investigations to be considered to exclude metastasis from choroid plexus carcinoma are MRI of spine to rule out leptomeningeal spread and also a cerebrospinal fluid cytology.

The histopathology of choroid plexus papilloma and choroid plexus carcinoma are difficult to be distinguished. Differences are looked at the appearances and histopathology characteristic [11]. Gross pathology will usually show a friable papillary like or cauliflower like appearance. Meanwhile, presence of increase in tumor necrosis, mitotic activity and change in growth pattern are more likely to resemble choroid plexus carcinoma than papilloma [7]. Likewise, the resemblance with metastatic papillary tumor is also making the diagnosis of choroid plexus carcinoma more challenging. On immunohistochemistry, choroid plexus carcinoma will stain positive for cytokeratins and has variable expression for Vimentin, S100, transthyretin and glial fibrillary acidic protein.

Owing to its low incidence, guideline in managing choroid plexus carcinoma is not well established. Best treatment option is still gross total resection, with Bettegowda, et al. defining it as reduction of more than $75 \%$ in tumor size. Partial resection was defined as 25 $75 \%$ reduction in tumor size. Gross total resection is achieved in $40-75 \%$ in choroid plexus carcinoma as opposed to $95 \%$ in choroid plexus papilloma [11]. The difficulty resulting in partial resection is due to highly vascularized tumor in deep location making it prone to intraoperative hemorrhage. A review by Sampath et al. has seen that mean blood loss for total resection is $540 \mathrm{ml}$ as compared to $890 \mathrm{ml}$ with partial resection [12]. In contrast, they also found the incidence of tumor bed hematoma was higher in total resection, but extent of excision did not significantly correlate with tumor bed hematoma. Other complications that have been more commonly reported post operatively includes subdural collection (32 to 43\%), pneumocephalus (40\%), focal deficit (36\%) and persistent hydrocephalus requiring CSF diversion surgery more commonly in adult $[9,12]$.

Other therapeutic options have been used as adjunct to surgery includes chemotherapy and radiotherapy particularly in patients with residual tumor. These adjuvant treatments however are not suitable for the young age group of less than 3 year old. Wolff et al have conducted a meta analysis through literature review of 566 choroid plexus tumor cases through 1966 till 1998 to determine the treatment modality. Their conclusions were, surgery significantly improve prognosis and radiotherapy significantly give better survival in choroid plexus carcinoma. Only 8 cases of 22 choroid plexus carcinoma were given chemotherapy and responded, thus the impact of this treatment option could not be sufficiently analyzed to be statistically of value [3]. Nonetheless, Berrak, et al. conducted a meta analysis of 361 choroid plexus carcinoma and found out of those given chemotherapy, etoposide is the most effective agent.

Similar case has been reported by Lozier, et al. in a 68 year old lady with supratentorial extraventricular choroid plexus carcinoma. She successfully underwent gross total resection followed by adjuvant radiotherapy and Temozolamide. No recurrence or residual tumor noted at 44 months follow up [5]. The difference with this patient was that his Karnofsky performance scale was already poor prior to our surgery thus rendered him poor prognosis.

The outcome of choroid plexus tumor depending on 3 factors which are; choroid plexus carcinoma histopathology, location of tumor and extent of resection $[4,12,13]$. However Wolff et al predicted that location of tumor has no prognostic relevance as opposed to Berrak, et al. who found survival is poorest in infratentorial tumor in choroid plexus carcinoma. Mean survival documented for supratentorial tumor was $26.9 \%$ at 10 years and none for infratentorial tumor. In case of a relapse after primary treatment of choroid plexus carcinoma, it is a poor prognostic factor for survival. 5 year survival of choroid plexus carcinoma is estimated to be $25-30 \%$ in patient with gross total resection $[7,11]$ Menon, et al. calculated the survival in subtotal resection for choroid plexus carcinoma was 36 months from surgery and 58 months for gross total resection [9]. Thus we should at best aim for gross total resection or multiple stage resection to prevent complications. 


\section{Conclusion}

Choroid plexus carcinoma is extremely rare in adult and its frequency at ectopic sites such as in this case is only been reported few in the literature. This case poses a great challenge to us in term of diagnosis and management. Total surgical resection was limited due to tumor vascularity and brain swelling. In the future, we hope a proper guideline can be established.

\section{References}

1. Kishore S, Nei G, Meena H, Anuradha K, Ved Pathak P, Bansal KK (2012) Choroid plexus carcinoma in an adult. $J$ Neurosci Rural Pract 3: 71-73. [Crossref]

2. Haroun RI, Li KW, Carson BS, Brem H (2000) Primary tumors of choroid plexus. Contemporary neurosurgeryv22: 1-7.

3. Wolff JE, Sajedi M, Brant R, Coppes MJ, Egeler RM (2002) Choroid plexus tumours. Br J Cancer 87: 1086-1091. [Crossref]

4. Berrak SG, Liu DD, Wrede B, Wolff JE (2011) Which therapy works better in choroid plexus carcinomas?. 2011. J Neurooncol 103: 155-162. [Crossref]

5. Lozier AP, Arbaje YM, Scheithauer BW (2009) Scheithauer. Supratentoria, extraventricular choroid plexus carcinoma in an adult: Case report. Neurosurgery 65 : 816-817. [Crossref]
6. Bekiesinska-Figatowska M, Madzik J, Biejat A, Maldyk J, Duczkowska A (2009) Choroid plexus carcinoma of the spinal canal without cranial lesion. European Journal of Radiology Extra 72: 107-109.

7. Gopal P, Parker JR, Debski R, Parker JC Jr (2008) Choroid plexus carcinoma. Arch Pathol Lab Med 132: 1350-1354. [Crossref]

8. Wilhelm M, Hirsch W, Merkenschlager A, Stepan H, Geyer C, Kiess W (2012) A rare case of congenital choroid plexus carcinoma. Pediatr Hematol Oncol 29: 643-646. [Crossref]

9. Menon G, Nair SN, Baldawa SS, Rao RB, Krishnakumar KP, Gopalakrishnan CV (2010) Choroid plexus tumor: An institutional series of 25 patients. Neurol India 58: 429-435. [Crossref]

10. Wyatt SS, Price RA, Holthouse D, Elsaleh H (2001) Choroid plexus carcinoma in an adult. Australas Radiol 45: 369-371. [Crossref]

11. Maimone G, Ganau M, Nicassio N, Paterniti S (2013) Paratrigonal choroid plexus papilloma presenting with satellite multiple supra and infratentorial hemorrhages. Int $J$ Surg Case Rep 4: 239-242. [Crossref]

12. Sampath S, Nitin G, Yasha TC, Chandramouli BA, Devi BI, et al. (2008) Does choroid plexus tumour differ with age? Br J Neurosurg 22: 373-388. [Crossref]

13. Bettegowda C, Adogwa O, Mehta V, Chaichana KL, Weingart J, et al. (2012) Treatment of choroid plexus tumors: a 20 -year single institutional experience. J Neurosurg Pediatr 10: 398-405. [Crossref]

Copyright: (C2017 Azhani C. This is an open-access article distributed under the terms of the Creative Commons Attribution License, which permits unrestricted use, distribution, and reproduction in any medium, provided the original author and source are credited. 